keratopathy ( 2 studies) and tamoxifen use (2 studies). Sex (2 studies) and history of cataract surgery ( 2 studies) were not found to be predictors of toxicity. Only few studies performed regression analysis presenting odds and/or hazard ratios with confidence intervals.

Conclusion: The most recognised predictor of cardiac toxicity was co-administration with azithromycin. In ocular toxicity, commonly cited predictors included those already recognised by the RCOphth ${ }^{1}$, as well as cumulative dose, increased age, weight considerations, $\mathrm{HCQ}$ blood levels and keratopathy. Further research is warranted on better characterising predictors of cardiac and ocular toxicity in patients on $\mathrm{HCQ}$ and $\mathrm{CQ}$ therapy.

REFERENCES:

[1] The Royal College of Ophthalmologists. Hydroxychloroquine and Chloroquine Retinopathy: Recommendations on Monitoring [Internet]. The Royal College of Ophthalmologists; 2020 [cited 2021 Jan 25].

Disclosure of Interests: Natasha Citeroni: None declared, Sanketh Rampes: None declared, Pinky Kotecha: None declared, Jan Schoones: None declared, Elena Nikiphorou: None declared, James Galloway Speakers bureau: JG has received honoraria / speaker fees from Abbvie, BMS, Celgene, Chugai, Gilead, Janssen, Lilly, Novartis, Pfizer, Roche, Sanofi, Sobi and UCB.

DOI: 10.1136/annrheumdis-2021-eular.1178

\section{POS0663 SAFETY AND EFFICACY OF COMBINING METHOTREXATE AND LEFLUNOMIDE AMONG PATIENTS WITH INFLAMMATORY ARTHROPATHIES: FINDINGS FROM THE PRIME REGISTRY}

M. Haroon ${ }^{1}$, A. Ashraf ${ }^{2}$, H. J. Shaheen ${ }^{1}$, S. Asif ${ }^{1}$, S. Batool ${ }^{1}$, F. Hashmi ${ }^{1}$, S. Ullah ${ }^{1} .{ }^{1}$ Fatima Memorial Hospital \& FMH College of Medicine and Dentistry, Department of Rheumatology, Lahore, Pakistan; ${ }^{2} \mathrm{CMH}$ Lahore Medical College \& Institute of Dentistry, Medicine, Lahore, Pakistan

Background: Currently, conventional synthetic DMARDs (csDMARDs) are the most commonly prescribed drugs as first-line treatment for peripheral arthritis. In resource-constrained settings where biologic agents are not widely available, there are limited therapeutic options for patients with rheumatoid arthritis (RA) and seronegative inflammatory arthropathies refractory to other csDMARD therapies. Hence, in our practice, we are inclined to use combination of potent DMARDs after MTX failure, prior to considering biologic therapies. We believe that combination of DMARDs, especially combining MTX and Leflunomide (LEF) provides a potent and valuable low-cost treatment option. Efficacy of MTX and LEF is very well established, but there have been lot of concern as regards their combination use due to potential risk of hepatotoxicity.

Objectives: We aimed to review our inflammatory arthropathies cohort data especially examining the safety, efficacy and drug retention of the combination usage of MTX and Leflunomide. We addressed this question using real-world data from the PRIME registry.

Methods: This was a cross-sectional study conducted using data collected at the time of patient enrolment in the PRIME registry. The PRIME Registry is a large, independent, prospective, observational cohort initiated in October 2019 that comprises patients diagnosed with RA, SLE, PSA or AS by a rheumatologist, and is being actively followed up. IRB approval and informed consent was obtained. A number of clinical variables were recorded. Detailed history was gathered from every patient regarding their present and past medications usage. Questions were asked directly about the usage or otherwise of all available DMARDs and biologics. The duration of usage, any adverse events, or the reasons for discontinuation were recorded. Evaluation of disease activity and severity was made as per internationally agreed definitions.

Results: The data of 766 inflammatory arthritis patients $(R A=663, P s A=103)$ was reviewed. Among them, 241 patients $(R A=196, P s A=45)$ were using combination therapy of MTX and LEF (combo MTX+LEF) with mean age $42.3 \pm 6$ years; $42 \%$ male]. These patients had failed MTX or LEF monotherapy. Among these 241 patients, 49 patients were also on concomitant hydroxychloroquine therapy. It was noted that median drug retention of combo MTX+LEF therapy has been 9.5 months (IQR 6-16). Regarding any adverse events of combo MTX+LEF therapy, hepatotoxicity (ALT $\leq 3$ times the upper limit of normal) was noted among $15(6.2 \%)$ patients, hepatotoxicity (ALT $\geq 3$ times the upper limit of normal) was noted among $8(3.3 \%)$ patients, and troublesome gastrointestinal upset (nausea, or vomiting, or diarrhoea) in $3(1.2 \%)$. Overall, only $13(5.4 \%)$ patients had to discontinue this combo MTX+LEF therapy due to adverse events. Disease activity among combo MTX+LEF users was as follows: $64 \%(n=29)$ of PsA patients had achieved MDA; $42 \%(n=83)$ of RA cohort were in DAS28 remission, $46 \%(n=91)$ of RA patients were having DAS low disease activity.

Conclusion: Combination of MTX and LEF was well tolerated and had good drug retention time, with $94.6 \%$ of patients having ongoing treatment to date. In low-income countries, where bDMARD availability is limited, financial arguments significantly influence decision making process, and our data provides initial evidence that MTX and LEF combination therapy could be an effective treatment option
Disclosure of Interests: Muhammad Haroon Speakers bureau: Roche, Novartis, Grant/research support from: Abbvie, Pfizer, Arfa Ashraf: None declared, Hafiza Javeria Shaheen: None declared, Sadia Asif: None declared, Shabnam Batool: None declared, Farzana Hashmi: None declared, Saadat Ullah: None declared

DOI: 10.1136/annrheumdis-2021-eular.1272

\section{POS0664}

A MULTICENTER RANDOMIZED STUDY IN RHEUMATOID ARTHRITIS TO COMPARE IGURATIMOD, METHOTREXATE, OR COMBINATION: 52 WEEK EFFICACY AND SAFETY RESULTS OF THE SMILE TRIAL

F. Du ${ }^{1}$, J. Xu' ${ }^{2}$ X. $\mathrm{Li}^{3}$, Z. $\mathrm{Li}^{4}$, X. $\mathrm{Li}^{5}$, X. Zuo ${ }^{6}$, L. Bi ${ }^{7}$, D. Zhao ${ }^{8}$, M. Zhang ${ }^{9}$,

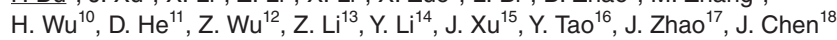
H. Zhang ${ }^{19}$, J. Li $i^{20}$, L. Jiang ${ }^{21}$, Z. Xiao ${ }^{22}$, Z. Chen ${ }^{23}$, G. Yin ${ }^{24}$, L. Gong ${ }^{25}$, G. Wang ${ }^{26}$, L. Dong ${ }^{27}$, W. Xiao ${ }^{28}$, C. Bao ${ }^{1} .{ }^{1}$ Renji Hospital of Shanghai Jiao Tong University School of Medicine, Department of Rheumatology, Shanghai, China; ${ }^{2}$ The First Affiliated Hospital of Anhui Medical University, Department of Rheumatology and Immunology, Hefei, China; ${ }^{3}$ The First Affiliated Hospital of USTC, Department of Rheumatology and Immunology, Hefei, China; ${ }^{4}$ Peking University People's Hospital, Department of Rheumatology and Immunology, Beijing, China; ${ }^{5}$ Qilu Hospital of Shandong University, Department of Rheumatology, Jinan, China; ${ }^{6}$ Xiangya Hospital Central South University, Department of Rheumatology and Immunology, Changsha, China $;{ }^{7}$ ChinaJapan Union Hospital of JiLin University, Department of Rheumatology and Immunology, Changchun, China; ${ }^{8}$ Changhai Hospital of Shanghai, Department of Rheumatology and Immunology, Shanghai, China; ${ }^{9}$ First Affiliated Hospital, Nanjing Medical University, Department of Rheumatology and Immunology, Nanjing, China; ${ }^{10}$ The Second Affiliated Hospital Zhejiang University School of Medicine, Department of Rheumatology and Immunology, Hangzhou, China; ${ }^{11}$ ShangHai Guang Hua Hospital of Integrated Traditional Chinese and Western Medicine, Department of Rheumatology, Shanghai, China; ${ }^{12}$ Xijing Hospital, Fourth Military Medical University, Department of Clinical Immunology, Xi'an, China; ${ }^{13}$ First Affiliated Hospital, Bengbu Medical College, Department of Rheumatology and Immunology, Bengbu, China; ${ }^{14}$ The Second Affiliated Hospital, Harbin Medical University, Department of Rheumatology and Immunology, Harbin, China; ${ }^{15}$ First Affiliated Hospital of Kunming Medical University, Department of Rheumatology and Immunology, Kunming, China; ${ }^{16}$ The Second Affiliated Hospital of Guangzhou Medical University, Department of Rheumatology and Immunology, Guangzhou, China; ${ }^{17}$ Jining No.1 People's Hospital, Department of Rheumatology and Immunology, Jining, China; ${ }^{18}$ The Second Xiangya Hospital of Central South University, Department of Rheumatology and Immunology, Changsha, China; ${ }^{19}$ The Third Xiangya Hospital of Central South University, Department of Nephropathy and Rheumatology, Changsha, China; ${ }^{20}$ Zhuzhou Central Hospital, Department of Rheumatology and Immunology, Zhuzhou, China: ${ }^{21}$ Fudan University Zhongshan Hospital, Department of Rheumatology and Immunology, Shanghai, China; ${ }^{22}$ The First Affiliated Hospital of Shantou University Medical College, Department of Rheumatology, Shantou, China; ${ }^{23}$ The First Affiliated Hospital of Soochow University, Department of Rheumatology, Suzhou, China; ${ }^{24}$ West China Hospital, Department of Rheumatology and Immunology, Chengdu, China; ${ }^{25}$ Tianiin Medical University General Hospital, Department of Rheumatology and Immunology, Tianjin, China; ${ }^{26}$ China-Japan Friendship Hospital, Department of Rheumatology and Immunology, Beijing, China;

${ }^{27}$ Tongji Hospital Tongji Medical College of HUST, Department of Rheumatology and Immunology, Wuhan, China; ${ }^{28}$ The First Hospital of China Medical University, Department of Rheumatology and Immunology, Shenyang, China

Background: Iguratimod (IGU) has demonstrated efficacy and safety for active rheumatoid arthritis (RA) patients in double-blind clinical trials in China and Japan as a new disease-modifying anti-rheumatic drug (DMARD). There are no studies evaluating the radiographic progression of structural joint damage of IGU for the treatment of RA using the mTSS as the primary endpoint.

Objectives: Our study was to evaluate the efficacy and safety of IGU monotherapy and IGU combined methotrexate (MTX) compared with MTX monotherapy, including the inhibitory effects of joint destruction.

Methods: This randomized, double-blind, parallel-controlled, multicenter study in patients with active RA who have not previously used MTX and biological DMARDs (bDMARDs) (ClinicalTrials.gov Identifier NCT01548001) was carried out in China. Patients were randomized 1:1:1 to receive IGU $25 \mathrm{mg}$ twice a day (bid), MTX 10mg once a week(qw) for the first 4 weeks and $15 \mathrm{mg}$ once a week(qw) for week 5 to 52 , or IGU combined MTX (IGU+MTX) for 52 weeks. The primary endpoints were to assess and compare American College of Rheumatology 20\% (ACR20) response and the change of modified total Sharp scoring (mTSS) score over 52 weeks (Intention-to-treat, ITT analysis). The non-inferiority test was used to analyze the difference of ACR20 response at 52 weeks between the IGU monotherapy and the MTX monotherapy arms, and the non-inferiority 
limit value was $10 \%$. The difference test was used for the comparison between the IGU+MTX and MTX monotherapy arms. Two-way ANOVA was used to analyze the difference of the changes of mTSS score of each arm compared with baseline value (0 week)

Results: A total of 895 patients were randomized to IGU $25 \mathrm{mg}$ bid ( $n=297$ ), MTX 10-15mg qw $(n=293)$, and IGU+MTX $(n=305)$. Baseline characteristics were comparable between the arms (Table 1)

Table 1. Demographic and Other Baseline Characteristics (SAS)

\begin{tabular}{lccc}
\hline & IGU & MTX & IGU+MTX \\
\hline Number of Subjects & $\mathbf{2 9 7}$ & $\mathbf{2 9 3}$ & $\mathbf{3 0 5}$ \\
\hline Age, mean (SD) years & $46.87(10.67)$ & $47.63(10.70)$ & $48.37(10.69)$ \\
Female/male, \% & $77.44 / 22.56$ & $79.18 / 20.82$ & $78.03 / 21.97$ \\
Duration of RA, mean(SD) years & $11.67 \pm 7.16$ & $11.60 \pm 7.98$ & $11.67 \pm 7.27$ \\
CRP, mean(SD) mg/L ${ }^{2}$ & $22.32 \pm 35.47$ & $20.67 \pm 26.61$ & $19.74 \pm 31.38$ \\
Tender joint count, mean (SD) & $14.59 \pm 9.16$ & $14.83 \pm 9.30$ & $14.93 \pm 9.88$ \\
Swollen joint count, mean (SD) & $9.81 \pm 6.63$ & $9.73 \pm 7.20$ & $9.51 \pm 6.22$ \\
DAS28-CRP, mean (SD) & $5.084 \pm 0.994$ & $5.102 \pm 0.979$ & $5.103 \pm 0.956$ \\
HAQ score, mean (SD) & $15.82 \pm 11.25$ & $15.24 \pm 10.93$ & $16.06 \pm 10.92$ \\
& & &
\end{tabular}

SAS: Safety Analysis Set; CRP: C-reactive protein;

DAS28: disease activity score; HAQ: Health Assessment Questionnaire

The study met its primary endpoints. More concretely, IGU monotherapy and IGU+MTX were found to be superior to MTX at week 52 with a higher ACR20 response of $77.44 \%(230 / 297, \mathrm{P}=0.0019)$ and $77.05 \%(235 / 305, \mathrm{P}=0.0028)$ versus $65.87 \%(193 / 293)$ (fig 1). As shown in fig 1

, the structural remission $(\Delta \mathrm{mTSS} \leq 0.5)$ was statistically significant for IGU monotherapy $(57.4 \%, \mathrm{P}=0.0308)$ but not for IGU+MTX arm $(55 \%)$ versus MTX monotherapy (47.8\%).

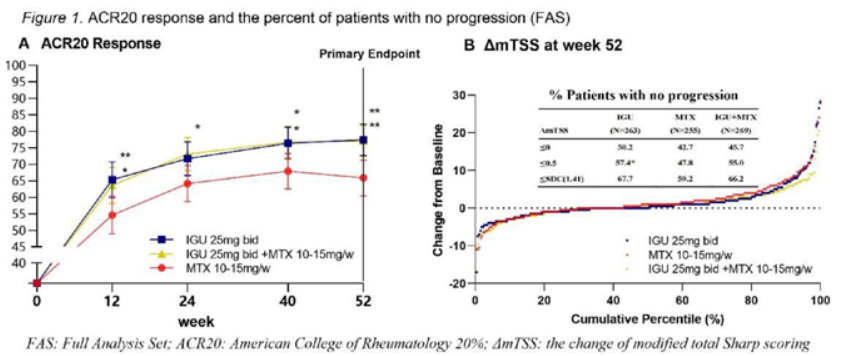

Overall incidence of the adverse events (AEs) leading to study discontinuation were reported in $13.8 \%(41 / 297)$ in IGU monotherapy arm, $11.26 \%(33 / 293)$ in MTX monotherapy arm and $11.51 \%(35 / 305)$ patients in IGU+MTX arm. The incidence of adverse drug reactions (ADR) leading to study discontinuation were $11.45 \%$ (34/297), $8.53 \%(25 / 293)$ and $9.21 \%$ (28/305), respectively. There was no one death and no significant difference in all the safety indicators among the three arms.

Conclusion: Iguratimod alone or in combination with MTX demonstrated superior efficacy with acceptable safety compared to MTX for patients with active RA who have not previously used MTX bDMARDs.

Disclosure of Interests: None declared

DOI: 10.1136/annrheumdis-2021-eular.1486

\section{\begin{tabular}{|l|l}
\hline POS0665 TRAJECTORIES OF GLUCOCORTICOID-THERAPY IN \\
\hline
\end{tabular} EARLY RHEUMATOID ARTHRITIS: FIRST RESULTS OF A SCOPING SYSTEMATIC REVIEW AND META- ANALYSIS OF OBSERVATIONAL COHORT STUDIES}

A. Palmowski ${ }^{1}$, F. Buttgereit ${ }^{1} .{ }^{1}$ Charité - Universitätsmedizin Berlin, Department of Rheumatology and Clinical Immunology, Berlin, Germany

Background: Glucocorticoids (GCs) are regularly used as a bridging therapy in early rheumatoid arthritis (eRA). As long-term treatment, especially at higher dosages, may lead to undesirable adverse events, GCs should be tapered as rapidly as clinically feasible.

Objectives: To assess real-world trajectories of GC-therapy initiated in patients with eRA and in methotrexate-naïve RA patients.

Methods: We conducted a scoping search in MEDLINE (via PubMed) to find articles (years 2005 - 2020) reporting on eRA (or methotrexate-naïve RA) patients from observational cohorts who start or take GCs at baseline. Articles had to describe either dosages or proportions of patients who took GCs or were able to taper GCs at two (minimum) pre-specified time points. The articles were screened by one reviewer (AP). Random-effects meta-analyses pooled results per outcome and time point if $\geq 3$ studies were available. $R$ software with package metafor was used for statistical analyses. A research protocol was published with protocols.io (10.17504/protocols.io.bpyfmptn).

Results: Our highly specific search strategy yielded 165 results. Twelve articles on nine cohorts were finally included. Eight cohorts originated in Europe, one in Africa. At baseline, about half of the patients with eRA were prescribed GCs with a mean dosage of $8 \mathrm{mg} / \mathrm{d}$ prednisone equivalent (fig 1

). Over time, both the proportion taking GCs and the mean dosage declined. There was substantial heterogeneity between studies.

Conclusion: Our results indicate that GCs remain regularly used drugs in eRA patients and in methotrexate-naïve patients with RA. While about $40 \%$ of patients still receive GCs after 24 months, mean dosages were tapered to "low" dosages $(\leq 7.5 \mathrm{mg} / \mathrm{d} \text { prednisone equivalent })^{1}$ in all cohorts that reported respective data Heterogeneity might be caused by country-specific differences. Unfortunately, the validity of sensitivity analyses would be poor due to the paucity of published data regarding GC dosages and proportions of patients taking GCs in observational RA cohorts. Major limitations of this scoping review are the very specific (and consequently less sensitive) search strategy and that the screening was conducted by one reviewer only.

\section{REFERENCES:}

[1] Buttgereit F, Da Silva JAP, Boers M, et al. Standardised nomenclature for glucocorticoid dosages and glucocorticoid treatment regimens: current questions and tentative answers in rheumatology. Ann Rheum Dis 2002;61(8):718-22. doi: 10.1136/ard.61.8.718
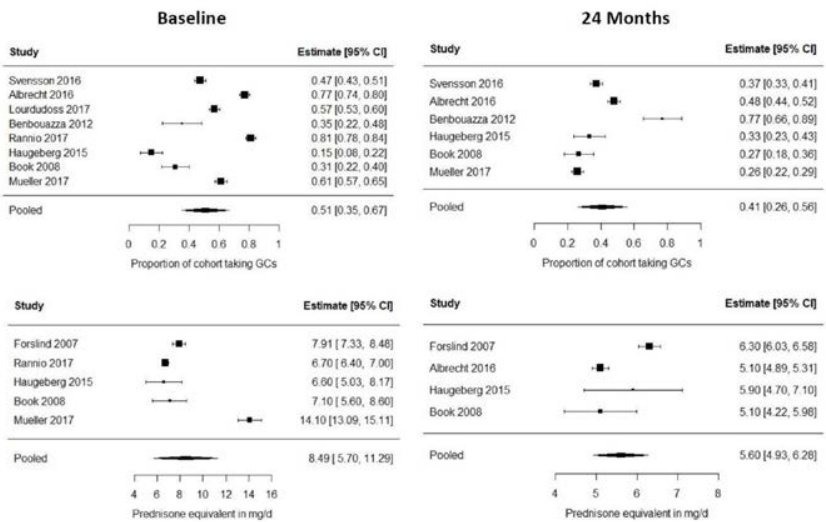

Figure 1. Meta-analyses of proportions taking glucocorticoids and mean dosages at baseline and 24 months. GCs: Glucocorticoids; Cl: Confidence interval.

Disclosure of Interests: None declared

DOI: 10.1136/annrheumdis-2021-eular.1526

\section{POS0666 TREATMENT EFFECTIVENESS OF UPADACITINIB AT 3 MONTHS IN US PATIENTS WITH RHEUMATOID ARTHRITIS FROM THE UNITED RHEUMATOLOGY NORMALIZED INTEGRATED COMMUNITY EVIDENCE (NICE[TM]) REAL-WORLD DATA}

A. Gibofsky ${ }^{1}$, B. Dhillon ${ }^{2}$, M. E. Pearson ${ }^{3}$, N. Tundia ${ }^{4}$, Y. Song ${ }^{4}$, K. Dunlap ${ }^{4}$, G. Wright ${ }^{5}$. ${ }^{1}$ Weill Cornell Medical College, Hospital for Special Surgery, New York, United States of America; ${ }^{2}$ United Rheumatology, Rheumatology, Hauppauge, United States of America; ${ }^{3}$ West Suburban Center for Arthritis, Rheumatology, Brookfield, United States of America; ${ }^{4}$ AbbVie Inc, Immunology, North Chicago, United States of America; ${ }^{5}$ Association of Women in Rheumatology, Rheumatology, New York, United States of America

Background: Upadacitinib (UPA), an oral Janus kinase inhibitor (JAKi), has demonstrated efficacy in the phase 3 SELECT clinical program, conducted across a range of patients (pts) with rheumatoid arthritis (RA). ${ }^{1-6}$ Real-world data for UPA, including in pts previously treated with a JAKi, have not yet been reported since global approvals beginning in 2019 .

Objectives: To assess the characteristics of US-based pts receiving UPA and its effectiveness in clinical practice at 3 months.

Methods: This observational study included US-based pts from the United Rheumatology Normalized Integrated Community Evidence (UR-NICE) database who initiated UPA $15 \mathrm{mg}$ once daily from FDA approval (August 2019) to July 31, 2020 and had $\geq 6$-month pre-baseline data available. Effectiveness was assessed in pts with a reported Clinical Disease Activity Index (CDAI) score at 3 months after UPA initiation and included proportions of pts achieving CDAI remission ( $\leq 2.8)$, CDAl low disease activity $(\leq 10)$, other disease activity measures, and pt-reported outcomes. A subgroup analysis assessed UPA effectiveness in pts with or without prior tofacitinib (TOFA) treatment. 